\title{
The messy biological basis of culture
}

\section{Adrian Woolfson heralds Antonio Damasio's bold argument that emotions define us.}

$\mathrm{I}$ $\mathrm{n}$ attempts to define what makes us uniquely human, emotions and feelings are often marginalized. These deeply ingrained, often irrational aspects of our behaviour seem destined to be the poor cousins of the rational cognitive functions that enable the formulation of mathematical theorems or operatic scores. In his bold and important book The Strange Order of Things, neuroscientist Antonio Damasio argues that in underestimating the contributions of such 'lower-level' brain phenomena to 'higherlevel' cognitive functions, science might have been missing out on some important biology. Similarly, neuroscience's emphasis on the origins of language as a shaper of culture might have eclipsed the role of feelings.

Damasio suggests that our hard-wired drives, urges, compulsions, impulses and automatic responses, such as hunger, desire and pain, originate from "subjective experiences of the momentary state of homeostasis" - that is, the body's routine, humdrum regulation of its visceral function. $\mathrm{He}$ argues that there is an organic dialogue between this biological process and the feelings that arise from our continuous scrutiny of it. That diverse penumbra of feelings and impulses, in turn, continuously infuses conscious thought and, ultimately, drives human behaviour.

Human nature is thus distilled from a delicate and protracted negotiation between the beating drums of instinct, shaped by core biological functions, and the attempts of conscious minds to negotiate culturally appropriate outcomes of its mandates. These negotiations are prone to failure, causing dissonance. Damasio theorizes that this generates the profusion of contradictions that both define humans as a species and emerge in our cultural artefacts - from the first stone tools to the Taj Mahal.

Damasio, by unseating the mind from its elevated throne within the brain, delivers an onslaught on one of the core dogmas of conventional neuroscience. In his view, mind is distributed for instance, to distant anatomical regions such as the peripheral neural networks that control organ function. Thus, different

of Things: Life, Feeling, and the Making of Cultures ANTONIO DAMASIO

Pantheon: 2018.

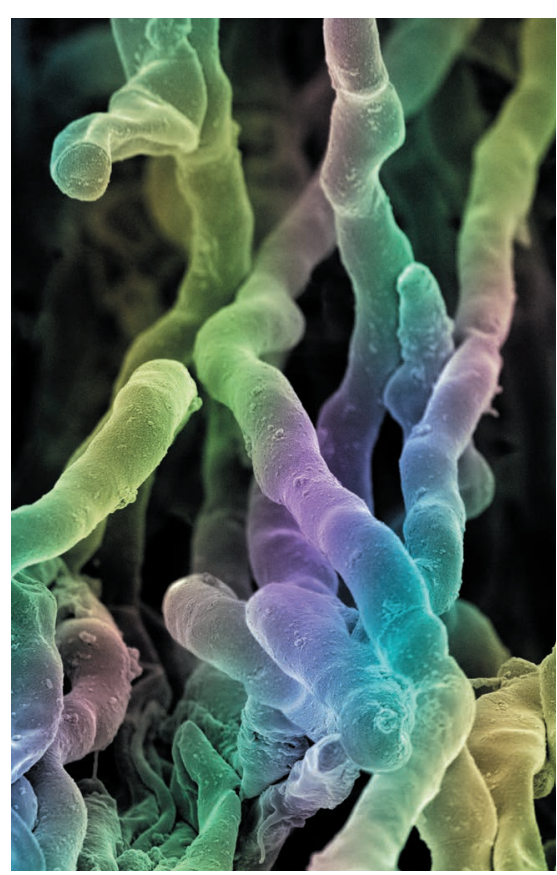

Streptomyces coelicoflavus bacteria (false colour).

tissues in the body contribute incrementally to the mind's function. Damasio's vision offers a new and specific incarnation of the thesis of unified body and mind.

The implications are fascinating. They suggest, for example, that organisms without complex neural structures might possess feelings, and even rudimentary minds. Damasio, incidentally, dates the origin of feelings to the emergence of the first nervous systems, around 600 million years ago.

And at a time when artificial intelligence and machine learning are rapidly advancing and seem poised to disrupt human hegemony, Damasio robustly challenges a belief cherished by transhumanists, who aspire to augment human capacities artificially. That belief is that the myriad aspects of human nature can be captured in their entirety and formalized in the notation of substrate-independent algorithms. A computer program such as Google's AlphaZero can currently master the totality of chess history in around four hours: the time it takes, approximately, to drive from New York City to Washington DC. But, if Damasio is correct, a computer lacking the benefits of biological hardware will never experience the subjective sensation of satisfaction that accompanies a human grandmaster's victory.

Damasio traces core components of the human "cultural mind", such as social $\vec{a}$ behaviour and cooperation, back to the nonhuman biology of unicellular organisms present at the inception of life. Bacteria do not sit up at night to contemplate the nature of their existence, and are unable to calculate the trajectories of distant planets. Nevertheless, they are in full command of an impressive repertoire of social behaviours. For example, when nutrients are scarce, bacteria eschew their hermit-like lives and clump together. They can also align into defensive palisades that can confer resistance to antibiotics.

Some of these genetically specified collective behaviours, Damasio argues, may be perceived as the precursors of "moral attitude". For instance, bacteria are able to identify kin through the nature of the molecules they synthesize, and seem to snub "cheaters" that breach social norms. According to Damasio, such behaviours, which provide a basis for social group formation and the emergence of cooperative behaviours, formed the basis for primitive cognition that was later elaborated to generate conscious minds.

Although compelling and refreshingly original, Damasio's thesis would have benefited from a more detailed exposition of the scientific evidence supporting his assertions. The lack of substantial discussion of supporting literature is a significant weakness. As a result, the text reads more like a nineteenth-century philosophical treatise than a contemporary study.

Still, The Strange Order of Things addresses several important questions. One of the more compelling consequences of Damasio's organic linkage of mental processes to the properties of biological hardware is that tinkering - such as the use of artificial amino acids and other synthetic-biology technologies - might have unforeseen consequences. Indeed, if Damasio is correct, the precise nature of the substrate that facilitates the performance of biological 'computing machines' will crucially determine their ability to generate mental phenomena. We certainly couldn't expect putative extraterrestrial life forms, which might have evolved by way of unfamiliar biological chemistries and structures, to experience feelings and mental processes in the same way that we do.
Adrian Woolfson is the author of Life Without Genes. e-mail:adrianwoolfson@yahoo.com 